\title{
Alteração do crescimento e dos teores de nutrientes com utilização de fertilizante organomineral em cenoura ${ }^{1}$
}

\author{
Jéssica Fernandes Kaseker ${ }^{2}$, Marília Camotti Bastos ${ }^{3}$, Rangel Consalter ${ }^{4}$, Átila Francisco Mógor ${ }^{5}$ \\ http://dx.doi.org/10.1590/0034-737X201461060011
}

\section{RESUMO}

$\mathrm{O}$ uso de fertilizantes organominerais contendo extratos de algas é recente no Brasil. Em vista disso, este estudo teve como objetivo avaliar o efeito da aplicação foliar de fertilizante organomineral contendo extrato da alga Ascophyllum nodosum (Activo $\left.{ }^{\circledR}\right)$ no desenvolvimento e nos teores de nutrientes em cenoura. O experimento foi conduzido na área de olericultura da Universidade Federal do Paraná, no município de Pinhais, PR, no ano de 2009, em sistema orgânico, utilizando cultivar do tipo Nantes. O delineamento experimental foi inteiramente casualizado, e os tratamentos consistiram na aplicação de fertilizante organomineral à base de extrato de algas. Foi utilizado um esquema fatorial com duas doses ( 0,2 e $0,4 \%)$ e duas épocas de aplicação (25 e 45 dias após o plantio), e um tratamento testemunha. As aplicações aumentaram a altura da parte aérea, o diâmetro radicular e o número de folhas, sendo esta variável também influenciada pela concentração. A produtividade aumentou com a aplicação do fertilizante organomineral, independentemente da concentração e da época de aplicação. Os teores nutricionais na parte aérea e nas raízes também foram alterados em decorrência das aplicações, sendo observada diminuição dos teores foliares de $\mathrm{Cu}$ e dos teores radiculares de $\mathrm{N}, \mathrm{Ca}, \mathrm{Mg}, \mathrm{Cu}$ e $\mathrm{Zn}$, possivelmente em função de efeito de diluição, pelo incremento da biomassa. Conclui-se que a aplicação do fertilizante organomineral contendo extrato de algas foi benéfica ao crescimento, desenvolvimento e produção das plantas de cenoura, o que resultou em diminuição na concentração de $\mathrm{N}, \mathrm{Ca}, \mathrm{Mg}, \mathrm{Cu}$ e $\mathrm{Zn}$ na raiz e de $\mathrm{Cu}$ na parte aérea.

Palavras-chave: Ascophyllum nodosum, organomineral, cultivo orgânico.

\section{ABSTRACT}

\section{Change in growth and nutrient content in carrots with the use of biofertilizer}

The use of organo-mineral biofertilizers containing alga extracts is recent in Brazil. Thus, the objective of this study was to evaluate the effect of leaf application of Ascophyllum nodosum extract biofertilizer (Activo®) on growth and on the nutrient content of carrot. The experiment was carried out in the garden area at Paraná Federal University, in the city of Pinhais, State of Paraná, in 2009, in an organic system, using the cultivar Nantes. The treatments consisted in the application of organo-mineral biofertilizer containing alga extract in different seasons and at different concentrations. The treatments were as follows: $0.2 \%$ on the $25^{\text {th }}$ day after planting (DAP); $0.4 \%$ on the $25^{\text {th }} \mathrm{DAP} ; 0.2 \%$ on the $45^{\text {th }} \mathrm{DAP}$ and $0.4 \%$ on the $45^{\text {th }} \mathrm{DAP}$. The applications increased shoot height, root diameter and number of leaves, which was also influenced by the concentration. Yield increased with the application of the

\footnotetext{
Recebido para publicação em 10/07/2013 e aprovado em 20/02/2014

${ }^{1}$ Trabalho desenvolvido na Universidade Federal do Paraná.

${ }^{2}$ Engenheira-Agrônoma, Mestre. Departamento de Solos e Recursos Naturais, Universidade do Estado de Santa Catarina, Avenida Luís de Camões 2090, Conta Dinheiro, 88520-000, Lages, Santa Catarina, Brasil. jessikaseker@ hotmail.com (autora para correspondência)

${ }^{3}$ Engenheira-Agrônoma, Mestre. Departamento de Solos, Universidade Federal de Santa Maria, Camobi, 97105-900, Santa Maria, Rio Grande do Sul, Brasil. mcamotti@ hotmail.com ${ }^{4}$ Engenheiro-Agrônomo, Mestre. Departamento de Solos e Engenharia Agrícola, Universidade Federal do Paraná, Setor de Ciências Agrárias, Rua dos funcionários, 1540, 80065-050, Curitiba, Paraná, Brasil. rangelconsalter@hotmail.com.br

${ }^{5}$ Engenheiro-Agrônomo, Pós-Doutor. Departamento de Fitotecnia e Fitossanitarismo, Universidade Federal do Paraná, Setor de Ciências Agrárias, Rua dos funcionários, 1540, 80065050, Curitiba, Paraná, Brasil. afmogor@gmail.com
} 
biofertilizer, regardless of concentration and period of application. The nutritional content in shoots and roots were also changed due to the application, where a decrease on foliar contents of $\mathrm{Cu}$ and on the root contents of $\mathrm{N}, \mathrm{Ca}$, $\mathrm{Mg}, \mathrm{Cu}$ and $\mathrm{Zn}$ was observed possibly due to the dilution effect as a result of the increased biomass. It is concluded that the application of organo-mineral fertilizer containing alga extract was beneficial to plant growth, development and yield of carrot plants, which resulted in a decrease in the nutritional contents.

Key words:Ascophyllum nodosum, biofertilizer, organic farming.

\section{INTRODUÇÃO}

Os agricultores investem em grandes quantidades de adubos minerais com o propósito de aumentar a produtividade e melhorar a qualidade de seus produtos. Este insumo muitas vezes é o mais caro na produção de olerícolas (Bruno et al., 2007), e pode ser substituído pela aplicação de fertilizantes orgânicos com resultados satisfatórios. A agricultura orgânica é ainda pouco expressiva no Brasil, porém sua relevância é crescente, tendo em vista que a agricultura convencional pode trazer impactos ambientais negativos (Mazzoleni \& Nogueira, 2006).

A cenoura (Daucus carota L.), espécie de maior importância econômica da família Apiaceae, é uma olerícola cultivada em todo o país, ocupando o quarto lugar em volume comercializado entre as olerícolas (Puiatti et al., 2007). A aplicação de fertilizantes orgânicos na cenoura apresenta bons resultados, especialmente se o solo apresentar baixa fertilidade (Souza $e t$ al., 2008). Segundo Negrini \& Melo (2007), a aplicação de composto orgânico como fonte de matéria orgânica foi suficiente para se obter produção de cenouras comparável à convencional.

Alguns estudos mostram que a aplicação de fertilizantes organominerais, aliada à adubação orgânica, apresenta melhora da produtividade e dos parâmetros morfológicos, como comprimento e diâmetro de raízes (Bruno et al., 2007; Oliveira et al., 2007), além de melhorar, de maneira geral, a concentração de nutrientes nas raízes (Pedrosa et al., 2007).

Estudos avaliando a eficiência de produtos à base de extrato de algas, notadamente da alga Ascophyllum nodosum, foram realizados com diversas culturas, entre elas couve (Silva et al., 2012), batata (Bettoni et al., 2008; Bardiviesso et al., 2011), alface (Limberger \& Gheller, 2012), feijão (Mógor et al., 2008) e maracujazeiro (Oliveira et al., 2011). Na maior parte desses trabalhos, os resultados obtidos foram positivos, indicando aumento do crescimento e da produção.

Os resíduos de frutas e olerícolas são, geralmente, desprezados pela indústria e poderiam ser utilizados como fontes alternativas de nutrientes (Pereira et al.,
2003). Por isso, vê-se a importância de se conhecer o teor nutricional, não apenas das raízes da cenoura, mas também de suas folhas.

Neste trabalho, o objetivo foi determinar a influência da aplicação foliar de fertilizante organomineral contendo extrato da alga Ascophyllum nodosum, no crescimento e nos teores de nutrientes de plantas de cenoura, utilizando-se diferentes concentrações e épocas de aplicação.

\section{MATERIAL E MÉTODOS}

O experimento foi conduzido em canteiro com 60 $\mathrm{m}^{2}$, localizados na área de olericultura do Centro de estações experimentais do Canguiri, da Universidade Federal do Paraná, no município de Pinhais, PR. O clima na região é classificado com $\mathrm{Cfb}$, segundo Koppen. $\mathrm{O}$ solo, classificado como Latossolo Vermelho-Amarelo álico (EMBRAPA, 2013) de textura argilosa, apresentou as seguintes características químicas: $\mathrm{pH}\left(\mathrm{CaCl}_{2}\right)=$ 6,$1 ; \mathrm{Al}^{+3}=0 ; \mathrm{H}+\mathrm{Al}=3,7 \mathrm{cmol}_{\mathrm{c}} \mathrm{dm}^{-3} ; \mathrm{Ca}^{2+}=7,2 \mathrm{cmol}_{\mathrm{c}}$ $\mathrm{dm}^{-3} ; \mathrm{Mg}^{2+}=3,4 \mathrm{cmol}_{\mathrm{c}} \mathrm{dm}^{-3} ; \mathrm{K}^{+}=1,44 \mathrm{cmol}_{\mathrm{c}} \mathrm{dm}^{-3} ; \mathrm{P}=$ $158,4 \mathrm{mg} \mathrm{dm}^{-3} ;$ Carbono orgânico $=37,4 \mathrm{~g} \mathrm{dm}^{-3} ; \mathrm{V} \%=$ 76; e CTC $=15,74 \mathrm{cmol}_{\mathrm{c}} \mathrm{dm}^{-3}$.

$\mathrm{O}$ canteiro foi preparado com rotoencanteirador e adubado com o equivalente a $20 \mathrm{tha}^{-1}$ de composto orgânico e $800 \mathrm{~kg} \mathrm{ha}^{-1}$ de termofosfato. Duas semanas após a adubação, em março de 2009, foi realizada a semeadura, em cinco linhas, com espaçamento de $20 \mathrm{~cm}$. Foram utilizadas aproximadamente $20 \mathrm{~g}$ de sementes, de cultivar do tipo Nantes, calibrada média, marca Isla ${ }^{\circledR}$.

Passados 20 dias após a semeadura, foi realizado o raleio, estabelecendo-se o espaçamento de aproximadamente sete centímetros entre plantas. Após o raleio, a área foi subdividida em 20 parcelas, sendo cinco tratamentos com quatro repetições. O delineamento experimental utilizado foi inteiramente casualizado. Os tratamentos foram referentes à aplicação de fertilizante organomineral (Activo ${ }^{\circledR}$ da empresa Átria, suspensão homogênea com densidade 1,3 , contendo $6 \%$ de carbono orgânico, $10 \%$ de agente complexante extrato da alga Ascophyllum nodosun, 0,25\% de agente complexante ácido cítrico, $5 \%$ de $\mathrm{Ca}$ e $9 \%$ de $\mathrm{K}_{2} \mathrm{O}$ ). Foi utilizado um 
esquema fatorial com duas doses e duas épocas de aplicação, sendo: testemunha; dose de 0,2 \%, aos 25 dias após o plantio (0,2 \% 25 DAP); dose de 0,4\%, aos 25 dias após o plantio; (0,4\% 25 DAP); dose de $0,2 \%$, aos 45 dias após o plantio; (0,2 \% 45 DAP) e dose de 0,4 \%, aos 45 dias após o plantio (0,4\% 45 DAP). A aplicação foi feita por via foliar, com pressão constante e vazão de $200 \mathrm{~L} \mathrm{ha}^{-1}$.

Durante o desenvolvimento das plantas, foram realizadas coletas aos 60, 75 e 100 dias após o plantio, sendo coletadas cinco plantas de cada parcela, e medidos o comprimento e diâmetro das raízes, com régua e paquímetro, comprimento da parte aérea e número de folhas. Na última avaliação, a parte aérea e as raízes das plantas coletadas de cada parcela foram pesadas, formando uma amostra, para obtenção do peso fresco, e, após secagem em estufa, a $65^{\circ} \mathrm{C}$, para obtenção do peso seco. Nestas amostras, também se realizou a análise química de folhas e raízes. Os teores de $\mathrm{P}, \mathrm{K}, \mathrm{Ca}, \mathrm{Mg}, \mathrm{Cu}, \mathrm{Mn}, \mathrm{Fe}, \mathrm{Zn}$ e Na foram analisados, após digestão total, a $500{ }^{\circ} \mathrm{C}$, e solubilização com HCl 3 mol L-1 (Martins \& Reissmann, 2007). A determinação do teor de $\mathrm{P}$ foi feita por colorimetria, em espectrofotômetro UV/VIS; K e Na foram determinados por fotometria de chama e, os demais elementos, por absorção atômica. Os teores de $\mathrm{N}$ foram obtidos pelo método de Kjeldahl.

As médias dos dados obtidos foram comparadas, utilizando-se análise de contrastes, por meio do teste de Scheffé, a $5 \%$ de probabilidade. Foram realizadas três comparações: efeito de aplicação, comparando-se a testemunha com os demais tratamentos (Contraste I); efeito de dose, comparando-se as duas doses aplicadas, sem considerar a época (Contraste II); e efeito de época, comparando-se as épocas de aplicação, sem considerar a dose utilizada (Contraste III).

\section{RESULTADOS E DISCUSSÃO}

\section{Atributos morfológicos}

Na primeira avaliação (60 DAP), ocorrida 15 dias após a segunda aplicação, foram observadas diferenças entre as doses aplicadas, sendo que a menor dose apresentou maior número de folhas (Tabela 1). Nas duas avaliações subsequentes (75 e 100 DAP), houve efeito de aplicação, sendo que as plantas que receberam pulverização apresentaram maior número de folhas, indicando que o fertilizante organomineral alterou a ontogenia das plantas ao longo do ciclo, promovendo o aumento do número de folhas, nas coletas nos períodos de 30 e de 55 dias após a segunda aplicação (45 DAP). Na última avaliação ocorreu efeito de dose, com a maior dose proporcionando maior número de folhas.
Em valores absolutos, a altura da parte aérea foi alterada positivamente pela aplicação do fertilizante organomineral nas três épocas de avaliação; contudo, a análise de contrastes indicou diferença significativa apenas na última avaliação, sem efeito da época ou da dose utilizada (Tabela 1).

Incrementos da parte aérea de plantas foram observados por Silva et al. (2012), sendo que a utilização do extrato de Ascophyllum nodosum em diferentes doses $(0,0 ; 0,2 ; 0,4$ e $0,6 \%)$ aumentou de forma linear o número de folhas em plantas de couve, e proporcionou aumento médio de $10 \%$ no comprimento da parte aérea, em relação ao da testemunha. Da mesma forma, Oliveira et al. (2011) observaram incremento da parte aérea de mudas de maracujazeiro-amarelo e atribuíram este efeito à presença de citocinina, na forma natural da alga $A$. nodosum, resultando em incremento da divisão celular, mesmo com pequenas concentrações, influenciando diretamente a altura da planta. Embora a parte aérea da cenoura não tenha importância comercial, exceto quando a comercialização ocorre em maços, o bom desenvolvimento das folhas aumenta a área fotossintética, o que pode resultar em maior crescimento radicular.

Em relação ao crescimento radicular, o comprimento médio não foi afetado pela aplicação do fertilizante organomineral (Contraste I). Contudo, na segunda avaliação foi observado maior comprimento radicular na menor dose (Contraste II), comportamento semelhante ao do número de folhas, na primeira avaliação (Tabela 1). Tendo-se em vista o hábito de crescimento da cenoura, era esperado que os tratamentos aplicados aos 25 DAP tivessem maior influência no crescimento em comprimento, porém não foi observado efeito de época, em nenhuma das avaliações (Contraste III). O diâmetro de raiz foi afetado pela aplicação do produto, independentemente da dose, ou época, apenas na terceira avaliação. A diferenciação comportamental observada entre diâmetro de raiz e os outros atributos morfológicos avaliados (comprimento de raiz, altura de parte aérea e número de folhas) indica que o meristema primário é mais sensível à aplicação de Activo®.

Assim como neste estudo, Bruno et al. (2007) observaram, além do aumento da parte aérea, que o diâmetro radicular da cenoura foi favorecido com a utilização de composto orgânico, juntamente com a aplicação de biofertilizante por via foliar, em comparação com o propiciado por outras fontes de adubação; entretanto, os efeitos também puderam ser observados no comprimento radicular.

Considerando-se o tamanho da raiz na ocasião da colheita (100 DAP), para o comprimento, as amostras se enquadrariam na classe 18 , que abrange cenouras com 18 a $22 \mathrm{~cm}$. Quanto ao diâmetro, sem a aplicação do 
fertilizante organomineral, as raízes se enquadrariam no calibre 2 (20 a $30 \mathrm{~mm}$ ); já, com a aplicação, a classificação seria calibre 3 (30 a 40 mm) (HORTIBRAS, 2009). Considerando-se que o consumidor brasileiro tem preferência por raízes de cenoura com comprimento e diâmetro variando, respectivamente, entre 15 e $20 \mathrm{~cm}$ e 30 e $40 \mathrm{~mm}$ (Vieira \& Pessoa, 2008), a aplicação melhora a aceitação do produto no mercado.

\section{Produção vegetal}

A aplicação do fertilizante organomineral, independentemente da época ou da dose, proporcionou aumentos da produtividade de parte aérea e de raízes de cenoura (Tabela 2). Nos tratamentos que receberam pulverização, a massa fresca (MF) da parte aérea das plantas foi da ordem de $52 \%$ superior à do tratamento testemunha. Essa diferença foi de $46 \%$, para a massa seca (MS) de parte aérea, de $40 \%$, para MF de raízes, e de 37 $\%$, para MS de raízes.

\section{Teor de nutrientes na parte aérea e raízes}

Na parte aérea, foi observado efeito da aplicação apenas dos teores de $\mathrm{Cu}$, que também foram influenciados pela dose utilizada, sendo que houve diminuição dos teores com a aplicação dos tratamentos e com o aumento da dose (Tabela 3). O teor de $\mathrm{N}$ apresentou diferença considerável entre as doses, também com diminuição do teor em função do aumento da dose. Considerandose que a parte aérea das plantas que receberam aplicação do fertilizante, foi superior à da testemunha (Tabelas $1 \mathrm{e}$ 2), infere-se que esteja ocorrendo efeito de diluição do teor dos nutrientes, já que representa relação de concentração na biomassa.

Nas raízes, os efeitos da aplicação sobre os nutrientes foram maiores, influenciando os teores de $\mathrm{N}, \mathrm{Ca}$, $\mathrm{Mg}$, Cu e Zn (Tabela 3). Em todos os casos houve redução dos teores nos tratamentos com aplicação, em relação aos da testemunha, o que novamente pode ser entendido como efeito de diluição, já que as cenouras desses tratamentos apresentaram maior massa seca (Tabela 2). Os teores de $\mathrm{Ca}$ e de $\mathrm{Cu}$ foram também influenciados pela época de aplicação, sendo que a aplicação mais tardia proporcionou as maiores reduções.

Apesar do observado decréscimo da concentração de alguns nutrientes (N, Ca, Mg, Cu e Zn) nas raízes de cenoura, os teores verificados neste estudo, mesmo no tratamento testemunha, foram inferiores aos observados na literatura, para a espécie (Sediyama et al., 1998; Pedrosa et al., 2007).

Tabela 1. Valores médios dos grupos contrastados para as variáveis número de folhas, altura da parte aérea, comprimento e diâmetro radicular, aos 60, 75 e 100 dias após o plantio (DAP)

\begin{tabular}{|c|c|c|c|c|c|c|c|c|c|c|c|c|}
\hline \multirow[b]{3}{*}{ DAP } & \multirow{2}{*}{\multicolumn{3}{|c|}{$N^{0}$ de folhas }} & \multirow{2}{*}{\multicolumn{3}{|c|}{$\frac{\text { Altura da parte aérea }}{\mathrm{cm}}$}} & \multirow{2}{*}{\multicolumn{3}{|c|}{$\frac{\text { Comprimento radicular }}{\mathrm{cm}}$}} & \multirow{2}{*}{\multicolumn{3}{|c|}{$\begin{array}{c}\text { Diâmetro radicular } \\
\mathrm{mm}\end{array}$}} \\
\hline & & & & & & & & & & & & \\
\hline & 60 & 75 & 100 & 60 & 75 & 100 & 60 & 75 & 100 & 60 & 75 & 100 \\
\hline \multirow[t]{2}{*}{ Contraste I } & $7,6^{\text {ns }}$ & $8,0^{* *}$ & $7,9^{*}$ & $24,7^{\text {ns }}$ & $26,6^{\mathrm{ns}}$ & $29,3^{*}$ & $13,8^{\text {ns }}$ & $17,4^{\mathrm{ns}}$ & $18,3^{\text {ns }}$ & $11,2^{\text {ns }}$ & $19^{\text {ns }}$ & $26,8^{* * *}$ \\
\hline & 8,3 & 9,1 & 9,5 & 26,1 & 29,1 & 34,8 & 14,7 & 17,4 & 19,5 & 13,1 & 19,5 & 30,6 \\
\hline \multirow[t]{2}{*}{ Contraste II } & $8,7^{*}$ & $9,1^{\mathrm{ns}}$ & $8,7^{* *}$ & $25,9^{\mathrm{ns}}$ & $29,5^{\text {ns }}$ & $34,1^{\mathrm{ns}}$ & $15,1^{\mathrm{ns}}$ & $17,8^{*}$ & $19,5^{\mathrm{ns}}$ & $13^{\mathrm{ns}}$ & $19,5^{\mathrm{ns}}$ & $30^{\mathrm{ns}}$ \\
\hline & 7,9 & 9,1 & 10,3 & 26,3 & 28,6 & 35,6 & 14,4 & 16,9 & 19,4 & 13,1 & 19,5 & 31,2 \\
\hline \multirow[t]{2}{*}{ Contraste III } & $8,3^{\mathrm{ns}}$ & $8,9^{\text {ns }}$ & $9,7^{\mathrm{ns}}$ & $25,9^{\text {ns }}$ & $29,2^{\mathrm{ns}}$ & $34,1^{\mathrm{ns}}$ & $14,1^{\mathrm{ns}}$ & $17,0^{\mathrm{ns}}$ & $19,0^{\mathrm{ns}}$ & $12,7^{\mathrm{ns}}$ & $19,0^{\mathrm{ns}}$ & $30,2^{\mathrm{ns}}$ \\
\hline & 8,3 & 9,3 & 9,2 & 26,3 & 28,9 & 35,6 & 15,4 & 17,7 & 19,9 & 13,4 & 20 & 31 \\
\hline
\end{tabular}

Na primeira linha, as médias da testemunha, dose de $0,2 \%$ e época de aplicação 25 DAP para os contrastes I, II e III, respectivamente; na segunda linha, as médias dos tratamentos com aplicação, dose de $0,4 \%$ e aplicação 45 DAP, respectivamente para os contrastes I, II e III. **= significativo a $5 \%$, *= significativo a $10 \%$, ns= não significativo pelo teste de Scheffé a $5 \%$ de probabilidade.

Tabela 2. Valores médios de massa seca e fresca de raiz (R) e parte aérea (PA) aos 100 dias após o plantio nos grupos contrastados

\begin{tabular}{|c|c|c|c|c|}
\hline & Massa Fresca PA & Massa Seca PA & Massa Fresca $\mathbf{R}$ & Massa Seca $R$ \\
\hline & \multicolumn{4}{|c|}{ g } \\
\hline \multirow{2}{*}{ Contraste I } & $63,8^{*}$ & $8,7^{*}$ & $260,9^{* *}$ & $25,7^{* * *}$ \\
\hline & 96,9 & 12,7 & 365,3 & 35,2 \\
\hline \multirow{2}{*}{ Contraste II } & 86,2 ns & $13,1^{\mathrm{ns}}$ & $363,2^{\mathrm{ns}}$ & $35,2^{\mathrm{ns}}$ \\
\hline & 107,6 & 12,4 & 367,4 & 35,1 \\
\hline \multirow{2}{*}{ Contraste III } & $90,9^{\text {ns }}$ & $12,8^{\mathrm{ns}}$ & $358,3^{\mathrm{ns}}$ & $34,3^{\mathrm{ns}}$ \\
\hline & 102,9 & 12,7 & 372,2 & 36,1 \\
\hline
\end{tabular}

PA=Parte aérea; $\mathrm{R}=$ raiz . Na primeira linha, as médias da testemunha, dose de $0,2 \%$ e época de aplicação 25 DAP para os contrastes I, II e III, respectivamente; na segunda linha, as médias dos tratamentos com aplicação, dose de $0,4 \%$ e aplicação 45 DAP, respectivamente para os contrastes I, II e III. **= significativo a 5\%, *= significativo a 10\%, ns= não significativo pelo teste de Scheffé a 5\% de probabilidade. 
Tabela 3. Teores médios de macro e micronutrientes nas raízes e parte aérea dos grupos contrastados

\begin{tabular}{|c|c|c|c|c|c|c|c|c|c|c|}
\hline & $\mathbf{N}$ & $\mathbf{P}$ & $\begin{array}{c}\mathrm{K} \\
\mathrm{kg}^{-1} \mathrm{MS}\end{array}$ & $\mathrm{Ca}$ & $\mathrm{Mg}$ & $\mathrm{Fe}$ & $\mathrm{Cu}$ & $\begin{array}{c}\mathrm{Zn} \\
\mathrm{mg} \mathrm{kg^{-1 }} \mathrm{M}\end{array}$ & Mn & $\mathbf{N a}$ \\
\hline \multicolumn{11}{|c|}{ Parte Aérea } \\
\hline Contraste I & $\begin{array}{l}27,4^{\mathrm{ns}} \\
27,7\end{array}$ & $\begin{array}{l}2,4^{\mathrm{ns}} \\
2,6\end{array}$ & $\begin{array}{l}28,6^{\mathrm{ns}} \\
30,8\end{array}$ & $\begin{array}{l}13,5^{\mathrm{ns}} \\
12,3\end{array}$ & $\begin{array}{l}5,4^{\mathrm{ns}} \\
5,4\end{array}$ & $\begin{array}{l}482,5^{\mathrm{ns}} \\
378,5\end{array}$ & $\begin{array}{l}6,7^{*} \\
5,2\end{array}$ & $\begin{array}{l}20,7^{\mathrm{ns}} \\
24,0\end{array}$ & $\begin{array}{l}37,7^{\mathrm{ns}} \\
32,8\end{array}$ & $\begin{array}{l}3619,6^{\mathrm{ns}} \\
3152,9\end{array}$ \\
\hline Contraste II & $\begin{array}{l}30,6^{*} \\
24,8\end{array}$ & $\begin{array}{l}2,6^{\mathrm{ns}} \\
2,6\end{array}$ & $\begin{array}{l}29,6^{\mathrm{ns}} \\
32,0\end{array}$ & $\begin{array}{l}11,8^{\mathrm{ns}} \\
12,9\end{array}$ & $\begin{array}{l}5,2^{\mathrm{ns}} \\
5,6\end{array}$ & $\begin{array}{l}434,3^{\text {ns }} \\
322,7\end{array}$ & $\begin{array}{l}6,0^{*} \\
4,5\end{array}$ & $\begin{array}{l}27,5^{\mathrm{ns}} \\
20,5\end{array}$ & $\begin{array}{l}31,5^{\mathrm{ns}} \\
34,1\end{array}$ & $\begin{array}{l}3685,6^{\mathrm{ns}} \\
2620,3\end{array}$ \\
\hline Contraste III & $\begin{array}{l}26,6^{\text {ns }} \\
28,8\end{array}$ & $\begin{array}{l}2,6^{\mathrm{ns}} \\
2,6\end{array}$ & $\begin{array}{l}29,7^{\text {ns }} \\
31,8\end{array}$ & $\begin{array}{l}12,7^{\text {ns }} \\
12,0\end{array}$ & $\begin{array}{l}5,8^{\mathrm{ns}} \\
5,32\end{array}$ & $\begin{array}{l}366,7^{\text {ns }} \\
390,3\end{array}$ & $\begin{array}{l}5,7^{\mathrm{ns}} \\
4,7\end{array}$ & $\begin{array}{l}27,1^{\mathrm{ns}} \\
20,9\end{array}$ & $\begin{array}{l}35,2^{\text {ns }} \\
30,4\end{array}$ & $\begin{array}{l}2934,1^{\text {ns }} \\
3371,7\end{array}$ \\
\hline \multicolumn{11}{|c|}{ Raiz } \\
\hline Contraste I & $\begin{array}{l}18,5^{* *} \\
13,7\end{array}$ & $\begin{array}{l}2,9^{\mathrm{ns}} \\
2,7\end{array}$ & $\begin{array}{l}20,1^{\mathrm{ns}} \\
21,2\end{array}$ & $\begin{array}{l}1,4^{* *} \\
1,1\end{array}$ & $\begin{array}{l}1,8^{* *} \\
1,6\end{array}$ & $\begin{array}{l}36,8^{\mathrm{ns}} \\
41,3\end{array}$ & $\begin{array}{l}6,5^{* * *} \\
4,9\end{array}$ & $\begin{array}{l}35,51^{*} \\
22,86\end{array}$ & $\begin{array}{l}6,3^{\text {ns }} \\
6,2\end{array}$ & $\begin{array}{l}1000,5^{\mathrm{ns}} \\
1061,8\end{array}$ \\
\hline Contraste II & $\begin{array}{l}14,0^{\text {ns }} \\
13,5\end{array}$ & $\begin{array}{l}2,7^{\mathrm{ns}} \\
2,8\end{array}$ & $\begin{array}{l}20,7^{\text {ns }} \\
21,8\end{array}$ & $\begin{array}{l}1,1^{\text {ns }} \\
1,2\end{array}$ & $\begin{array}{l}1,5^{\mathrm{ns}} \\
1,6\end{array}$ & $\begin{array}{l}36,1^{\text {ns }} \\
46,5\end{array}$ & $\begin{array}{l}4,9^{\mathrm{ns}} \\
4,9\end{array}$ & $\begin{array}{l}27,51^{\text {ns }} \\
18,22\end{array}$ & $\begin{array}{l}5,6^{\text {ns }} \\
6,7\end{array}$ & $\begin{array}{c}1125,5^{\text {ns }} \\
998,1\end{array}$ \\
\hline Contraste III & $\begin{array}{l}13,0^{\text {ns }} \\
14,5\end{array}$ & $\begin{array}{l}2,7^{\mathrm{ns}} \\
2,8\end{array}$ & $\begin{array}{l}20,9^{\text {ns }} \\
21,6\end{array}$ & $\begin{array}{l}1,3^{* *} \\
1,0\end{array}$ & $\begin{array}{l}1,6^{\mathrm{ns}} \\
1,5\end{array}$ & $\begin{array}{l}42,6^{\mathrm{ns}} \\
40,0\end{array}$ & $\begin{array}{l}5,4^{*} \\
4,4\end{array}$ & $\begin{array}{l}20,24^{\mathrm{ns}} \\
25,49\end{array}$ & $\begin{array}{l}6,5^{\mathrm{ns}} \\
5,9\end{array}$ & $\begin{array}{l}1000,1^{\text {ns }} \\
1123,6\end{array}$ \\
\hline
\end{tabular}

Na primeira linha, as médias da testemunha, dose de $0,2 \%$ e época de aplicação 25 DAP para os contrastes I, II e III, respectivamente; na segunda linha, as médias dos tratamentos com aplicação, dose de $0,4 \%$ e aplicação 45 DAP, respectivamente para os contrastes I, II e III. **= significativo a $5 \%$, *= significativo a $10 \%$, ns= não significativo pelo teste de Scheffé a $5 \%$ de probabilidade.

Comparando-se os teores médios foliares e radiculares (Tabela 3), nota-se que as folhas, que geralmente são desprezadas na alimentação humana, apresentam teores superiores aos encontrados nas raízes para a maioria dos nutrientes analisados, com destaque para o $\mathrm{Fe}$.

\section{CONCLUSÕES}

A aplicação de fertilizante organomineral a base de extrato de algas exerce efeito benéfico para a cultura da cenoura, proporcionando maior diâmetro radicular, maior desenvolvimento da parte aérea e maior produtividade, mesmo em solo com alta fertilidade. Contudo, neste estudo, o acentuado aumento da produção de matéria de seca de folhas e de raízes resultou em diminuição de teores nutricionais, em ambas as partes.

\section{REFERÊNCIAS}

Bardiviesso DM, Backes C, Bôas RLV, Santos AJM \& Lima CP (2011) Aplicação foliar de extrato de alga na cultura da batata. Horticultura brasileira, 29:S1170-S1177.

Bettoni MM, Adam WM \& Mógor AF (2008) Tuberização de batata em função da aplicação de extrato de alga e cobre. Horticultura brasileira, 26:S5256-S5260.

Bruno RLA, Viana JS, Silva VF, Bruno GB \& Moura MF (2007) Produção e qualidade de sementes e raízes de cenoura cultivada em solo com adubação orgânica e mineral. Horticultura Brasileira, 25:170-174.

EMBRAPA (2013) Sistema Brasileiro de Classificação de Solos, $3^{\text {a }}$ Edição. Brasília, Embrapa. 353 p.

HORTIBRAS - Instituto Brasileiro de Qualidade em Horticultura (2009) Norma Referente à Classificação de Cenoura para o Programa Brasileiro para Melhoria dos Padrões Comerciais e Embalagens de Hortigranjeiros. Disponível em: <http://www.hortibrasil.org.br/ classificacao/cenoura/arquivos/norma.html $>$. Acessado em: $13 \mathrm{de}$ junho de 2013.
Limberger PA \& Gheller JA (2012) Efeito da aplicação foliar de extrato de algas, aminoácidos e nutrientes via foliar na produtividade e qualidade de alface crespa. Revista Brasileira de Energias Renováveis, 1:148-161.

Martins AP \& Reissmann CB (2007) Material vegetal e as rotinas laboratoriais nos procedimentos químico-analíticos. Scientia Agrária, 8:01-17.

Mazzoleni EM \& Nogueira JM (2006) Agricultura orgânica: características básicas do seu produtor. Revista de Economia e Sociologia Rural, 44:263-293.

Mógor AF, Ono EO, Rodrigues JD \& Mógor G (2008) Aplicação foliar de extrato de alga, ácido L-glutâmico e cálcio em feijoeiro. Scientia Agraria 9:431-437.

Negrini ACA \& Melo PCT de (2007) Efeito de diferentes compostos e dosagens na produção de cenoura (Daucus carota L.) em cultivo orgânico. Revista Brasileira de Agroecologia, 2:1036-1039.

Oliveira AP de, Barbosa AHD, Cavalcante LF, Pereira WE \& Oliveira ANP de (2007) Produção da batata-doce adubada com esterco bovino e biofertilizantes. Ciência Agrotécnica, 31:1722-1728.

Oliveira LAA, Góes GB, Melo IGC, Costa ME \& Silva RM (2011) Uso do extrato de algas (Ascophyllum nodosum) na produção de mudas de maracujazeiro-amarelo. Revista Verde, 6:01-04.

Pedrosa MW, Sediyama MAN, Vidigal SM, Santos MR dos, Salgado LT \& Nobre M da CR (2007) Concentração de nutrientes em raízes de três cultivares de cenoura, adubadas com diferentes doses de compostos orgânicos. Revista Brasileira de Agroecologia, 2:1145-1149.

Pereira GIS, Pereira RGFA, Barcelos MFP \& De Morais AR (2003) Avaliação química da folha de cenoura visando ao seu aproveitamento na alimentação humana. Ciência agrotécnica, 27:852-857.

Puiatti M, Finger FL, Verzon M \& Paula Júnior TJ de (2007) Cenoura (Daucus carota L.). In: Venzon M \& Paula Júnior TJ de (Eds.) 101 Culturas - Manual de tecnologias agrícolas. Belo Horizonte, EPAMIG. p.253-262.

Sediyama MA, Vidigal SM, Pereira PRG, Garcia NCP \& De Lima PC (1998) Produção e composição mineral de cenoura adubada com resíduos orgânicos. Bragantia, 57:379-386. 
Silva CF, Garcia KGV, Silva RM, Oliveira LAA \& Tosta MS (2012) Desenvolvimento inicial de mudas de couve-folha em função do uso de extrato de alga (Ascophyllum nodosum). Revista Verde, 6:7-11.

Souza AF, Mesquita Filho MV \& Fontes RR (2008) Adubação. In: EMBRAPA CNPH - Centro Nacional de Pesquisa de Hortaliças. Sistemas de Produção, 5 - Versão Eletrônica. Disponível em: <http:// sistemasdeproducao.cnptia.embrapa.br/FontesHTML/Cenoura/ Cenoura_Daucus_Carota/adubacao.html $>$. Acessado em: 13 de junho de 2013
Vieira JV \& Pessoa HBSV (2008) Cultivares. In: EMBRAPA CNPH Centro Nacional de Pesquisa de Hortaliças. Sistemas de Produção, 5 - Versão Eletrônica. Disponível em: <http:// sistemasdeproducao.cnptia.embrapa.br/FontesHTML/Cenoura/ Cenoura_Daucus_Carota/cultivares.html>. Acessado em: 13 de junho de 2013. 